\title{
Emergomyces pasteuriana causing a disseminated infection in an immunocompromised patient
}

\author{
K.B. Gast ${ }^{1}$, J.W.J. van Esser ${ }^{2}$, P. Huizinga ${ }^{1}$, J.J. Verweij ${ }^{1}$, P.H.J. van Keulen ${ }^{3}$ \\ ${ }^{1}$ Laboratory for Medical Microbiology and Immunology, St Elisabeth Hospital, Tilburg, The Netherlands \\ 2 Department of Internal Medicine, Amphia Hospital, Breda, The Netherlands \\ ${ }^{3}$ Microvida, Amphia Hospital, Breda, The Netherlands
}

\section{Introduction}

Until recently the clinical relevance of Emmonsia species, which are saprophytic, dimorphic fungi, was limited to causing a very rare pulmonary disease (adiaspiromycosis) in immunocompetent patients. In 1994, a novel Emmonsia species, E. pasteuriana, was described causing a disseminated mycosis in a HIVinfected woman. Recently, this species has been renamed to Emergomyces pasteuriana. E. pasteuriana differs from the Emmonsia species, that cause adiaspiromycosis, as it produces no adiaspores and is different from a clinical perspective as it causes disseminated, often fatal infections in predominantly immunocompromised patients. We report an immunocompromised patient with a disseminated $E$. pasteuriana infection, who was successfully treated with posaconazole.

\section{Case}

A 62-year old women of Moroccan descent, with biliary cirrhosis, chronic kidney failure and auto-immune haemolytic anaemia treated with $50 \mathrm{mg} /$ day of prednisolone and a past history of large B cell non-Hodgkin's lymphoma, was presented to the emergency room with shortness of breath. Initial chest X-ray showed a density in the right upper lobe. Further analysis with positron emission tomography-computed tomography (PET-CT) was performed, showing two PET positive lesions in the right upper lobe and multiple PET positive subcutaneous lesions, suggestive of malignancy (Figure 1a). Biopsy of two subcutaneous lesions showed no malignant cancer cells, but infiltration of predominantly histiocytes and within the histiocytes infiltration of structures resembling yeast cells (Figure 2). Additional molecular fungal identification with internal transcribed spacer rRNA polymerase chain reaction (ITS PCR) revealed Emergomyces pasteuriana. Cultures of these subcutaneous lesions showed, after 12 days of incubation, growth of a dimorphic fungus (Figure 3). Molecular identification at the Center of Expertise in Mycology Radboudumc/CWZ (J. Meis, MD) confirmed Emergomyces pasteuriana and additional antifungal susceptibility was performed. The following MICs were determined ( $\mathrm{mg} / \mathrm{L}$ ): amphotericin B 0.031, anidulafungin 0.016, micafungin $<0.008$, itraconazole 0.063 , voriconazole 0.25 , posaconazole 0.063 , isavuconazole 1 and fluconazole 64. Patient was tested negative for human immunodeficiency virus infection and bone marrow biopsy showed no haematological malignancy. The patient was treated with posaconazole and prednisolone therapy was gradually lowered to $5 \mathrm{mg}$ per day. After 2 months of posaconazole treatment, she experienced nausea and posaconazole tablets were switched to an oral suspension. A PET-CT scan conducted after 6 months of therapy showed a decline in number and PET intensity of the subcutaneous and lung lesions (Figure 1b) and after 14 months of treatment all subcutaneous lesions and the two lung lesions disappeared (Figure 1c). Patient experienced no pulmonary symptoms and the posaconazole was stopped.

\section{Conclusion}

This is the first Dutch report of a disseminated Emergomyces pasteuriana infection in an immunocompromised patient who recovered on posaconazole treatment. Furthermore, this case underlines that clinicians should be aware of invasive fungal infections in immunocompromised patients as they may mimic malignancy. In this case, molecular diagnostic techniques could rapidly detect and identify fungi directly from clinical specimens.
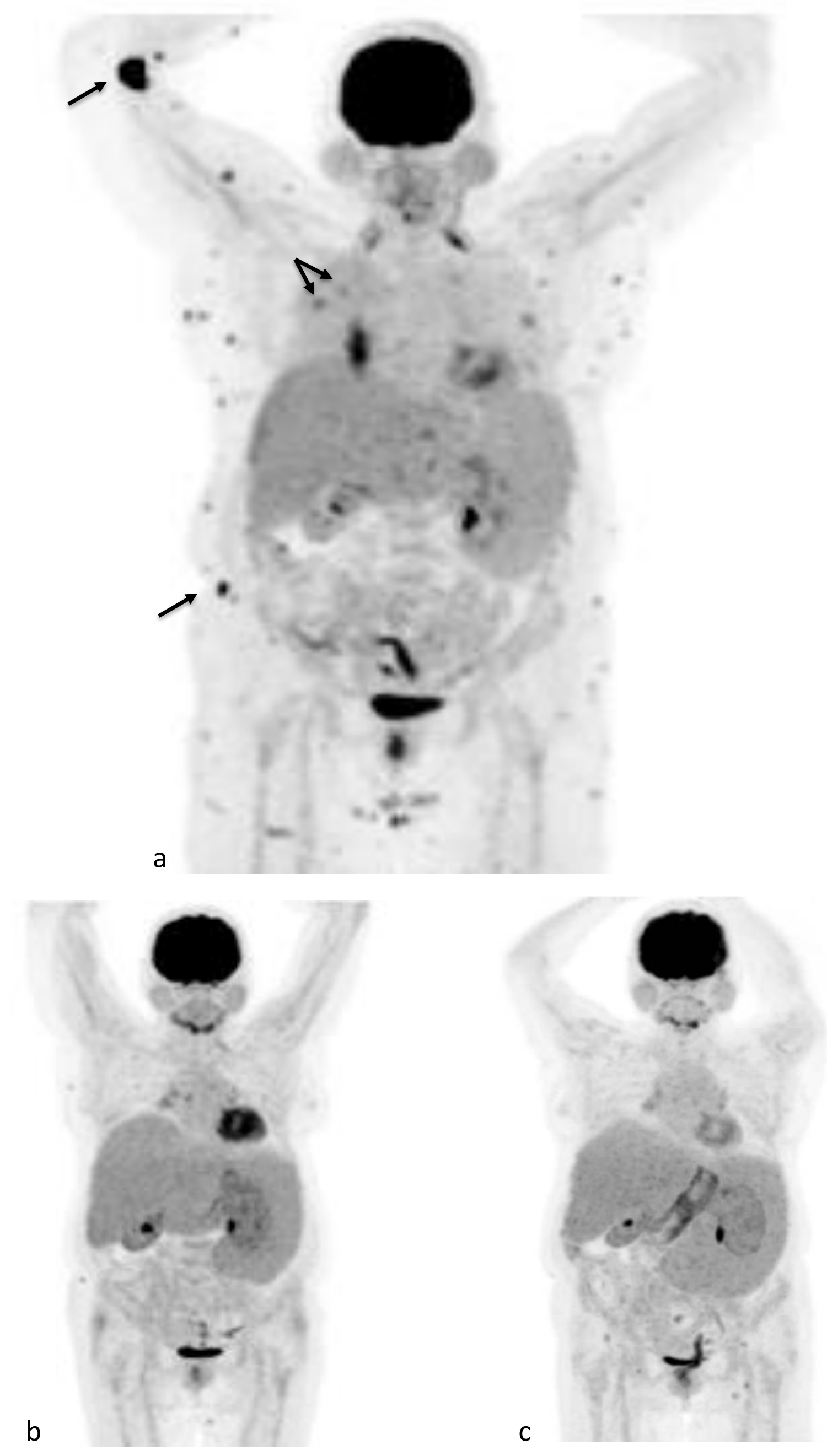

Figure 1. Position emission tomography-computed tomography images at initial presentation (panel a), after 6 months (panel b) and after 14 months (panel c) of posaconazole treatment. The arrows indicate the two lung lesions and the largest subcutaneous lesions.

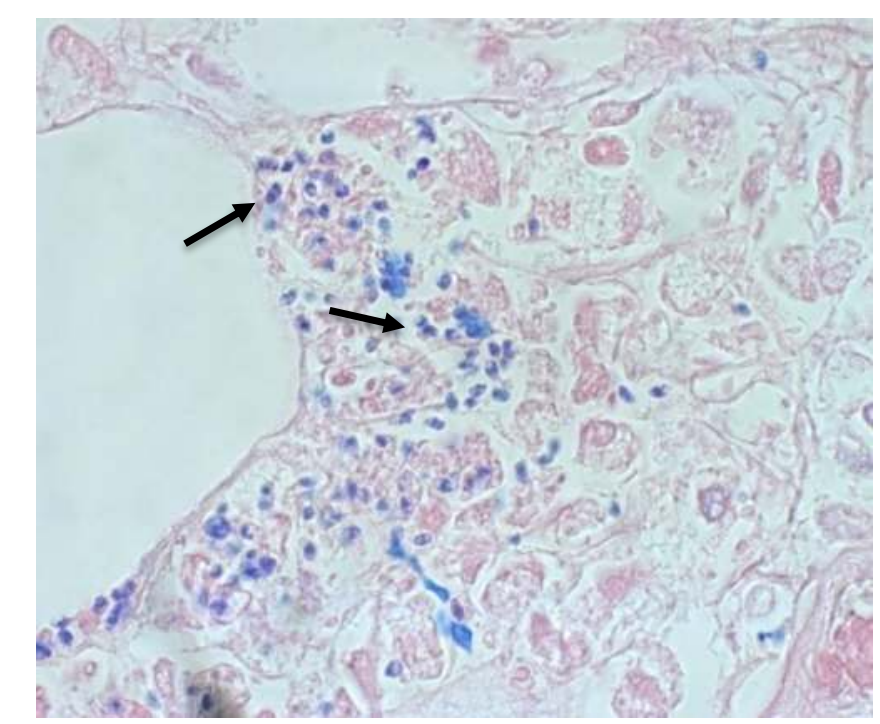

Figure 2. Histopathologic section (hematoxylin-eosin stain) of a subcutaneous lesion, showing yeastlike structures (arrows).

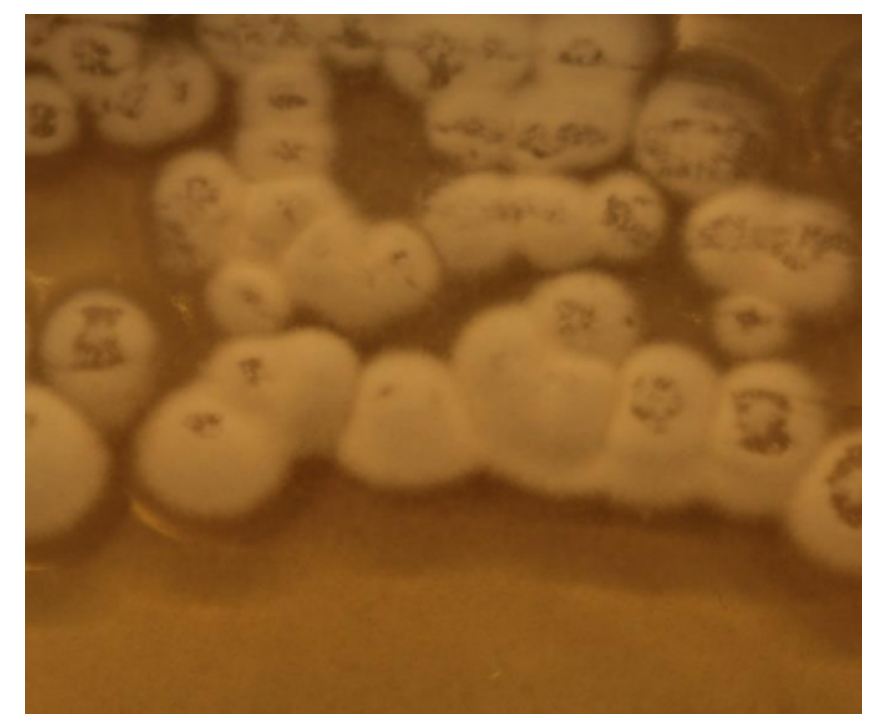

Figure 3. Colonial morphology of Emergomyces pasteuriana after 12 days incubation at $25^{\circ} \mathrm{C}$ on Sabouraud agar. 